\title{
EL LÉXICO DE ESPECIALIDAD EN EL SIGLO XIX: EL MANUAL DE METEOROLOGÍA POPULAR DE GUMERSINDO VICUÑA (MADRID, 1880)
}

\author{
M. Ángeles García Aranda \\ (Universidad Complutense de Madrid) \\ magaranda@filol.ucm.es
}

\begin{abstract}
Resumen
Este trabajo se ocupa del vocabulario científico que aparece en el Manual de Meteorologia popular de Gumersindo Vicuña (Madrid, 1880). Tras describir brevemente la situación que vive la ciencia en la segunda mitad del siglo XIX gracias a la entrada en la docencia universitaria de materias como las matemáticas o la física, a la creación de las Facultades de Ciencias y a la fundación de la Real Academia de Ciencias Exactas, Físicas y Naturales (1847), la Academia de Ciencias Morales y Políticas (1857) y la Real Academia Nacional de Medicina (1861), el artículo se centra en la descripción y análisis del vocabulario científico del Manual de Vicuña y en el tratamiento que este léxico recibe en el Diccionario de la Academia. Finalmente, se presenta la lista de voces y significados que se encuentran en la obra.

PALABRAS CLAVE: Lenguaje científico, Lexicografía del español, Meteorología, siglo XIX.
\end{abstract}

\begin{abstract}
This work studies the scientific vocabulary included in Gumersindo Vicuña's Mantual de Meteorología popular (Madrid, 1880). In particular, the paper focuses on its description and analysis in Vicuña's treatise and also examines how the Real Academia Española deals with this kind of lexicon in its Dictionary, after presenting briefly the status of the science in Spain during the second half of the $19^{\text {th }}$ Century, in constant improvement thanks to the establishment of several Science Faculties (Facultades de Ciencias) and the foundation of the Real Academia de Ciencias Exactas, Físicas y Naturales (1847), the Academia de Ciencias Morales y Políticas (1857) and the Real Academia Nacional de Medicina (1861). Finally, the scientific voices found at the treatise are listed together with their correspondent meanings.

KEYWORDS: Scientific language, Spanish Lexicography, Meteorology, $19^{\text {th }}$ century.
\end{abstract}

\section{Introducción}

El presente trabajo trata sobre el vocabulario científico que aparece en el Manual de Meteorologia popular de Gumersindo Vicuña, publicado en Madrid en 1880. Pero es imposible penetrar en el léxico de especialidad sin contextualizar antes las ciencias en la España decimonónica, pues es precisamente en la segunda mitad de esta centuria cuando sufren una significativa recuperación que, sin duda alguna, determinó la aparición de numerosos repertorios científicos y técnicos, la inclusión de abundantes tecnicismos en diccionarios generales y las traducciones de textos científicos fundamentales compuestos en otras de lenguas.

Así mismo, fue trascendental para el desarrollo de las ciencias en este periodo la labor de instruidos especialistas que con sus publicaciones, experimentos y conferencias divulgaron los avances científicos y pasaron a formar parte activa de la vida social y política de nuestro país. Uno de estos hombres fue Gumersindo Vicuña, catedrático de Física 
matemática de la Universidad Central y autor de varias obras sobre física, matemática, agricultura o meteorología que enriquecen estas disciplinas y las dan a conocer al público general para que así España mejorara y floreciera tras el crítico periodo histórico que le había tocado vivir.

\section{Bio-bibliografía del autor}

Gumersindo Vicuña y Lazcano nació en Portugalete el 13 de enero de 1840 y murió en 1890. Vivió, pues, en la segunda mitad del convulso siglo XIX, momento especialmente importante para la historia de las ciencias en España, ya que "los años siguientes a la revolución de 1868 significaron, en primer término, una liberación de la opresión ideológica a la que se había llegado durante la parte final del reinado de Isabel II [...]. En segundo lugar, el extremado liberalismo académico vigente durante estos años permitió la aparición de una amplia serie de instituciones científicas extraoficiales. Muchas de ellas carecieron de relieve o tuvieron una vida efímera, pero hubo algunas que ofrecieron una alternativa renovadora al anquilosado mundo académico oficial, que tuvo una considerable influencia en la elevación del nivel de la actividad científica española durante la Restauración" (López Piñero, 1992: 16-17).

La historia de la ciencia decimonónica está claramente dividida en dos etapas: la primera, que coincide con los primeros sesenta años de la centuria, ha sido denominada "periodo de catástrofe" por el freno que supusieron la guerra, los problemas sociopolíticos y la maltrecha economía a los avances iniciados en la Ilustración; si bien, hubo algunas excepciones en los años cuarenta y cincuenta, de manera que la escasa "actividad científica española se caracteriza por la dependencia del empeño de personas o de grupos muy limitados que, en ocasiones, llegan a conectar con la comunidad científica internacional y, en algunos casos, a influir en ella, pero que trabajan al margen de la sociedad en la que viven" (López Piñero, 1992: 16) ${ }^{1}$. En la segunda etapa, en cambio, se produce un florecimiento de la ciencia gracias a la entrada en la docencia universitaria de materias como las matemáticas o la física y a la creación de las Facultades de Ciencias, independientes ya de los estudios de Filosofía y Letras tal y como defendió la Ley Moyano de $1857^{2}$. Además, se crea la Real Academia de Ciencias Exactas, Físicas y Naturales (1847), la Academia de Ciencias Morales y Políticas (1857) y la Real Academia Nacional de Medicina (1861), cuyos miembros publican valiosos trabajos sobre temas diversos, imparten conferencias, se relacionan con científicos interna-

1 Una descripción parecida de este periodo histórico nos la ofrece Bertha Gutiérrez Rodilla en La ciencia empieza en la palabra (1998), en donde refleja la precaria situación de España con estas palabras: "El curso de la historia de la primera mitad de ese siglo en nuestro país no fue en absoluto favorable al desarrollo científico, pues el despegue industrial y técnico de los países europeos coincidió con un estancamiento en España" (pp. 80).

2 Los artículos 76 y 136 de la Ley de instrucción pública sancionada por S.M. en 9 de Septiembre (Madrid, Imprenta Nacional, 1957) dicen que: "Se estudiarán en las Facultades de Filosofía y Letras y en las de Ciencias, exactas, físicas y naturales, las materias pertenecientes a ellas que forman parte de otras facultades o carreras; y los estudios comunes a varias enseñanzas se harán en una misma cátedra, a no impedirlo la situación del establecimiento o el excesivo número de alumnos", y "Para el estudio y enseñanza de las ciencias exactas, fisica y naturales, en su mayor extensión, habrá en Madrid una Escuela Superior de Ciencias exactas, físicas y química, un Museo de Historia Natural y un Observatorio astronómico. Estas tres Escuelas reunidas constituyen la Facultad de Ciencias. Cada uno de estos establecimientos tendrá un local independiente, y un reglamento particular en que se dispondrán los estudios del modo que los mismos alumnos hagan frecuentes ejercicios prácticos de las asignaturas que cursen". 
cionales o difunden sus descubrimientos, lo que supone su penetración en la vida política, social y cultural del país. Para el desarrollo de la ciencia del siglo XIX son también importantes las Escuelas Especiales de Ingenieros, y así se fundan la de Ingenieros de Minas en 1835, la de Ingenieros de Montes en 1835, la de Ingenieros de Arquitectura en 1844 y la de Ingenieros Agrónomos en 1855; del mismo modo reabre sus puertas en 1835 otra institución fundamental para el progreso y divulgación de la ciencia, el Ateneo de Madrid, Científico, Literario y Artístico tras su cierre de 1823.

En este ambiente, en el que políticos y científicos se esmeraban por sacar a la ciencia española del letargo en que había caído por diversas razones históricas, se desarrolla la labor de Gumersindo Vicuña. Se doctora en Ciencias e Ingeniería Industrial, y se convierte en el primer catedrático de Física matemática de la Universidad Central, dirige la revista $L a$ Semana Industrial ${ }^{3}$, es presidente de la Asociación de Ingenieros Industriales, diputado al Congreso y compone una serie de obras que, merecidamente, lo convirtieron en uno de los científicos más importantes de la centuria.

Su producción aúna tanto los escritos teóricos destinados a sus colegas y especialistas, como los manuales divulgativos sobre física, matemática y sus beneficios para la vida cotidiana. Entre sus principales tratados podemos destacar: Teoría y cálculo de las máquinas de vapor y gas con arreglo a la termodinámica (Madrid, Manuel Tello, 1872) ${ }^{4}$, Motores empleados en la Industria (Madrid, Manuel Tello, 1872), Elementos de fisica al alcance de todo el mundo (Madrid, J. Noguera, 1874-1875), Progresos industriales (Madrid, Medina y Navarro, 1876), en colaboración con Nicolás M. ${ }^{a}$ Serrano el Tratado completo de Agricultura moderna. Enciclopedia de las teorias y buenas prácticas que debe conocer todo labrador, propietario rural, ganadero, hortelano, etc. con arreglo a los últimos adelantos, y puesta al alcance de todo linaje de personas (Madrid, M. Rodríguez editor,

3 Esta publicación es una de las muchas que vieron la luz en la segunda mitad del siglo XIX, periodo de gran auge para la prensa científica, y así aparecen, entre otros, los primeros números de la Revista minera (1850), la Revista de Obras Públicas (1853), El ingeniero industrial (1858), El agricultor español (1850-51), la Revista forestal, económica y agrícola (1868), La Mineria (1872), Industria e Invenciones (1872), El Petróleo (1873), Anales de la Construcción y de la Industria (1876), Anales de la Agricultura (1877), la Revista Tecnológica Industrial (1878), La Electricidad (1883), La industria ibérica (1884), La Ciencia eléctrica (1890), la Revista Agricola de la Asociación de Ingenieros Agrícolas (1892), El Progreso agricola y Pecuario (1895) o La industria vinicola (1899). Vid. E. Fernández Clemente, 2002.

4 En la revista La Ilustración española y americana: periódico de ciencias, artes, literatura, industria y conocimientos útiles (Madrid, 16 de diciembre de 1873, año XVII, $\mathrm{n}^{\circ} 47, \mathrm{pp} .746$ ) se publicó una reseña sobre este volumen, en la que podemos leer críticas como: "Esta obra explica menuda clara y metódicamente la teoría de las máquinas con arreglo a la Termodinámica, novísima ciencia que tan admirables y maravillosos resultados alcanza. La especial competencia del señor Vicuña para escribir semejante tratado, es tan superior, como natural y propia en quien, merced a sus conocimientos, posee títulos científicos de muy elevado linaje, junto con una cátedra dificilísima, ganada en brillante y ardua oposición [...]. Con esos elementos, y siguiendo un plan excelente, trata el señor Vicuña todas las cuestiones que a las teorías de las máquinas atañen, y logra exponer de una manera perfecta tan dificilísimo asunto, nuevo fuera, y más aun dentro de España. Publícase actualmente en alemán una obra monumental, que tendrá cuatro volúmenes, sobre la teoria de las máquinas, por el doctor Groshof, de la que hay repartida la entrega que comprende la teoría mecánica del calor. Comparando este trabajo, que sólo entienden profundos matemáticos, con el del señor Vicuña, por los sencillo y claro, es muy preferible la obra del catedrático de la Universidad matritense. Dicha obra con grabados, impresa con lujo, correcta, limpia y elegantemente por don Manuel Tello, ha logrado aplausos de personas muy entendidas. Cualquiera que intente conocer el asunto de que se trata, debe estudiar ese notable trabajo, que tanto honra al docto ingeniero y catedrático de Física-matemática de la Universidad de Madrid". 
1877), Manual de física popular (Madrid, Dirección y Administración, 1878) ${ }^{5}$, Impresiones y juicio de la Exposición Universal de 1878 (Madrid, Imprenta y Litografía de "La Guirnalda", 1878) o la Introducción a la teoría matemática de la electricidad (Madrid, Imprenta y Litografía de "La Guirnalda", 1883). Entre sus discursos sobresale el que leyó en la Universidad Central en el acto de apertura del curso académico 1875-1876 y que versó sobre el "Cultivo actual de las ciencias físico-matemáticas en España", en el que repasa la situación de estas disciplinas entre 1835 y 1875 . Además, se preocupó, como vasco que era, de Los fueros y sus defensas (Bilbao, Andrés P. Cardenal, 1897) y prologó el libro de Leonardo Torres Quevedo El sitio de Bilbao en 1874, en el que se describen las vicisitudes de este acontecimiento.

Sin duda alguna la labor científica de Gumersindo Vicuña, y su preocupación por las ciencias y sus utilidades, fueron las razones para nombrarlo, el 10 de junio de 1883, académico de número de la Real Academia de Ciencias Exactas, Físicas y Naturales. El tema que eligió para su discurso de recepción fue las "Relaciones principales entre las teorías matemáticas de la Física" (1883). La Academia de Ciencias fue fundada en 1847 por Antonio Zarco del Valle y tuvo entre sus primeros objetivos la confección de un Vocabulario Cientifico y Técnico que resolviese los numerosos problemas lingüísticos que habían surgido tras la abundante entrada de neologismos y voces nuevas procedentes de lenguas en las que el desarrollo de la ciencia y la técnica había originado la publicación de indispensables obras sobre estas materias.

En definitiva, Gumersindo Vicuña es uno de los científicos más importantes de la segunda mitad del siglo XIX que no se limitó a llevar a cabo su trabajo entre las paredes de un laboratorio, sino que se preocupó por darlo a conocer más allá del ámbito especialista para que todo aquel que quisiera se pudiera aprovechar de los avances de las ciencias.

\section{El Manual de Meteorología popular: estructura, contenido y objetivos}

A finales de 1878, Gumersindo Vicuña termina el Manual de Meteorología popular, lo entrega al editor en 1879 y ve la luz en 1880 (Madrid, Tipografía de G. Estrada) ${ }^{6}$.

La obra se divide en cuatro partes, a saber:

$\left.1^{a}\right)$ Fenómenos generales, compuesta de los capítulos "idea de la Meteorología" y "Repartición del calor";

$2^{a}$ ) Equilibro de la atmósfera o capítulos III, "la temperatura", IV, "la humedad", V, "los vientos", VI, "la presión", y VII "la electricidad";

$\left.3^{a}\right)$ Meteoros y magnetismo terrestre, que se reparte a su vez en "meteoros acuosos", "meteoros eléctricos", "meteoros luminosos" y "magnetismo terrestre", que corresponden, a su vez, a los capítulos VIII, IX, X y XI, respectivamente; y

$\left.4^{a}\right)$ Pronósticos del tiempo, es decir, "causas eficaces" o capítulo XII, "empleo de los instrumentos" o capítulo XIII y "clima de España" o capítulo XIV.

5 Esta obra ha sido utilizada por la Real Academia Española en el CORDE (Corpus diacrónico del español) como representante de la Física en la época contemporánea.

6 Esta información la proporciona el propio autor al final de la obra para justificar que no haya citado algunos de los tratados que se publicaron desde la composición del Manual hasta su publicación.

Manejamos el ejemplar con signatura 5/10889 de la Biblioteca Nacional de España. 
Se trata, pues, de un volumen que pretende recoger todos los fenómenos, instrumentos y teorías que se usan y afectan a la Meteorología, así como su utilidad para agricultores, navegantes y población general, propósitos que se repiten en diferentes momentos, y así Gumersindo Vicuña afirma, al tratar sobre los vientos, que pueden servir "para la orientación de las calles en las poblaciones, para situar los lugares infectos, como mataderos, cementerios, etc., en puntos tales, que las corrientes generales lleven las miasmas lejos de la población más próxima; ya para montar molinos de viento, ya para ciertas faenas agrícolas, ya, sobre todo, para la navegación" (págs. 103-104). De la misma manera, reconoce que "así como las lluvias limpian la atmósfera arrastrando las sustancias orgánicas que suele haber en su extensión, las nieblas, por el contrario, impiden el movimiento de estas sustancias y de las emanaciones pestilentes de algunas lagunas. Bajo este aspecto pueden ser perjudiciales para la salud pública, pero no por la humedad en sí misma, que a la generalidad de los seres vivos favorece más que perjudica" (págs. 146).

Por otro lado, el Manual de Meteorologia popular conjuga las explicaciones doctas y las exposiciones eruditas, propias de un físico, con aclaraciones y comentarios didácticos y más divulgativos, pues no olvida que la obra puede ser útil también para destinatarios menos formados y familiarizados con la ciencia teórica.

En este sentido, destaca la gran formación y los conocimientos de Gumersindo Vicuña, que le permiten: a) ser crítico con algunos meteorólogos, pues sostiene que el número de los resultados ozonométricos varía "con arreglo a la opinión y hasta el capricho de algunos meteorólogos" (págs. 142); b) formular quejas, como cuando afirma "cuanto llevamos dicho en este capítulo tiene por objeto principal llamar la atención de nuestros lectores sobre una materia poco cultivada, y que quizás llegue a adquirir con el tiempo más importancia de la que hoy posee en la esfera científica, porque, como se ha visto, no es fácil concretar los aparatos y elementos de este estudio, a no entrar en largas y enojosas disertaciones, o limitarse a las indicaciones de los aparatos usados hasta hace pocos años, sencillos pero imperfectos" $(140)$; c) desmentir leyendas, "de antiguo hay la creencia de que el relente es perjudicial para la salud, sobre todo en los climas cálidos, como el de la Isla de Cuba, creencia fundada en una observación continua; hoy se explica el hecho, o mejor dicho, sus consecuencias, por una condensación del vapor de la atmósfera, que al caer en menudísima lluvia arrastra consigo la materia orgánica flotante en el ambiente, y la hace depositar en el suelo, siendo absorbida por los pulmones de quien se pasea al aire libre en estos instantes y depositándose también sobre su piel" (págs. 153); d) reflexionar sobre los avances de la ciencia garantizando que "esta tarea [el estudio de borrascas, tormentas y tempestades] está hoy en vías de ejecución, y no es dudoso que dentro de pocos años se conocerá lo que hay de regular y periódico en estos fenómenos, que hoy parecen a las gentes incultas como inexplicables y completamente caprichosos" (págs. 167); e) contrastar diferentes hipótesis de trabajo, porque "entre las muchas explicaciones que se han dado del magnetismo terrestre, hay una muy en boga en nuestros días que consiste en atribuir este fenómeno, en sus variaciones, a las manchas que se observan en el sol" (págs. 185); f) poner en tela de juicio la "idea un tanto exagerada sobre la influencia que ejerce la luna en el tiempo" (págs. 198); o g) reconocer que "respecto de nuestra España queda aún mucho por hacer para conocer bien sus climas" (págs. 227).

Además, ejemplifica con Cristóbal Colón los servicios que hace la Meteorología a la navegación, cita los "modernos viajes en globo" (págs. 150), conoce diferentes tipos de barómetros (barómetro de Fortín, barómetro-modelo de Montsouris...), confiesa las limita- 
ciones de los resultados de la investigación de esta disciplina ${ }^{7}$, y continuamente muestra su sabiduría y su actualización bibliográfica, mencionando trabajos actuales sobre la materia y nombrando a especialistas en Meteorología.

A la vez, advierte que no profundiza en la materia demasiado porque el libro pretende ser divulgativo, y determinados aspectos de la ciencia son demasiado elevados para el público general; así, al tratar la teoría de los ciclones, reconoce que "sin entrar en grandes desarrollos para explicar todas y cada una de las fases del asunto indicado por el epigrafe, nos limitaremos a lo más esencial, conforme a la opinión de los mejores meteorólogos, puesto que nuestro libro es más bien expositivo que doctrinal" (págs. 96); del mismo modo, cuando habla de las curvas que surgen del barómetro en funcionamiento reconoce que "de esta suerte, y con un operador hábil y práctico en el asunto, se llega a traducir en múmeros el resultado de dichas curvas, empleando también ciertos procedimientos generales, que no podemos indicar en este librito, porque exigen conocimientos matemáticos para ser comprendidos" (págs. 123), finalmente, en el capítulo sobre Magnetismo terrestre confiesa que "en cuanto a la fuerza magnética, es preciso valerse de la aguja auxiliar $t$ y ayudarse de ciertas fórmulas y elementos que no posee la generalidad de las gentes a quienes dedicamos este manual, y el aparato en su conjunto exige cierta práctica y conocimientos en quien lo maneja" (págs. 179).

Por otro lado, y para que el contenido del Manual resulte claro y útil, recurre a comparaciones, ejemplos, cuadros y unas ilustraciones finales de algunos de los instrumentos utilizados en Meteorología ${ }^{8}$.

\section{El léxico del Manual de meteorología popular}

El vocabulario de especialidad en el siglo XIX ha sido, en las últimas décadas, objeto de varios estudios. Ahora bien, la mayoría de estos trabajos se ocupan del léxico de la fisica, de la química, de la política, de la enología, de la construcción, de la fotografía, del vino, de la minería, del ferrocarril, de la farmacia o de la medicina en diferentes textos y autores, así como de su recepción en el DRAE, su presencia en repertorios no académicos y de las traducciones del francés y del inglés que del léxico de especialidad se hicieron en esta centuria9.

7 Sirvan como ejemplos las siguientes palabras de Vicuña: "no entramos en detalles sobre esta rama [actinometría] por su novedad y poca generalización, así como por no haberse llegado a una unidad general y sencilla para su medición", en la página 53, o "Bajo el aspecto teórico hay grandes dudas hoy sobre los detalles y explicación completa de la producción de la electricidad atmosférica, aunque parece averiguado que se origina principalmente en las regiones intertropicales, sobre todo en las capas elevadas de la atmósfera", en la 134.

8 Vicuña es didáctico cuando relaciona el efecto de una tromba de agua con un embudo, cuando enumera los principales episodios de granizo, piedra y caída de meteoritos ocurridos en España, cuando ilustra y coteja diferentes cartas magnéticas, cuando resume en varias tablas la información sobre los vientos obtenida en los observatorios de la Península o cuando relaciona el clima español con las regiones agrícolas y aconseja los cultivos más adecuados para cada una de ellas.

9 Véanse, por ejemplo, el número 55 (2003) de la revista Asclepio sobre "Los textos en Ia mirada del filólogo y del cientifico", el volumen VII (2004) que la Revista de Investigación Lingüistica dedicó a "Lenguas técnicas y de especialidad" o trabajos como los de I. Ahumada (2000), M. Alvar Ezquerra (1989), D. Azorín e I. Santamaría (1998), F. Bajo Santiago (2002), P. Battaner (1977, 2001 y 2004), G. Claveria y J. Torruella (1998), N. Contreras Izquierdo (1997 y 1998), R. Gállego Paz (2002), C. Garriga (1996-1997, 1997, 1998 y 2004), C. González Orejón (2000), J. F. Guillén (1967), J. Gutiérrez Cuadrado (1996-1997, 1998 y 2004), B. M. autièrrez Rodilla (1999 y 2000) o L. Sala Caja (2001). 
Pocos han sido, sin embargo, los casos en los que los investigadores han extraído el léxico de una obra científica y lo han presentado, descrito y analizado para conocer así la forma en que los expertos decimonónicos en ciencia se enfrentaron a la explicación de términos técnicos y de especialidad ${ }^{10}$. En este sentido, el Manual de meteorología popular es un magnífico exponente, pues, como se trata de una obra popular y divulgativa, su autor aclara la mayoría de los términos científicos que salen en sus páginas ${ }^{11}$.

No olvidemos, sin embargo, que el vocabulario de especialidad incluido en el Manual de Meteorología popular de Gumersindo Vicuña no es un repertorio lingüístico, pues, como todos los diccionarios terminológicos, "se refiere a conceptos y realidades objetivamente estructuradas y, por tanto, su conocimiento depende del de esas realidades, no del de la lengua a la que pertenece" (Porto Dapena, 2002: 49); ahora bien, aunque la mayoría de las definiciones de Vicuña contienen explicaciones y detalles más propios de un artículo enciclopédico que de uno lingüístico, esto es, caracterizan la realidad no el significado de las voces, a veces las definiciones son muy similares a las que se encuentran en un diccionario de lengua, o ¿hay mucha diferencia entre "Strati. Nubes que forman capas paralelas al horizonte e inmediatas a él, predominando en la puesta del sol." de Vicuña y "Estrato. Meteor. Nube que se presenta en forma de faja en el horizonte" del repertorio académico?

Estas razones, junto a la atención que Vicuña presta a otras cuestiones lingüísticas interesantes, son las que explican el por qué de este trabajo.

Así, por ejemplo, el autor proporciona la etimología de la voz "monzón" y afirma que: "como vientos periódicos anuales, podemos designar los monzones, nombre que se deriva de una voz árabe que significa estación; ya hemos citado los de la India, y su dirección es bastante regular en cada estación. En el Atlántico, y en la región ocupada entre las calmas tropicales y las ecuatoriales, hay también monzones, aunque no tan patentes como los de la India, cuyas mesetas del Himalaya, que se calientan mucho en el verano, originan el fenómeno" (págs. 91), o la de "isoterma", de la que sostiene que "su nombre, de raíces griegas, quiere decir de igual temperatura" (págs. 56). Y también desvela el origen de alguna palabra como en "los vientos etesios del Mediterráneo, nombre que le daban los griegos son verdaderos monzones" (págs. 92).

Otras veces se plantea la idoneidad de algunas denominaciones y llega a asegurar que "aquí es el lugar de decir algo sobre las mareas atmosféricas, si es lícita esa palabra" (págs. 126).

De la misma manera, acota la acepción de algunas voces a un campo de especialidad o terminológico concreto, como ilustra el artículo de la voz "tornado", cuya definición alude exclusivamente al ámbito de la navegación: "nombre con que nuestros navegantes designan las grandes trombas, originadas por la tendencia a la rotación en los alisios en la zona que pudiéramos llamar clásica de las tormentas, o sea en la ecuatorial" (págs. 166).

$\mathrm{Y}$ en alguna ocasión llaman la atención las designaciones que emplea, y las definiciones que propone. Así, en la clasificación de los vientos - basada en la presión por metro cuadrado, la velocidad por hora y la velocidad por segundo-, distingue: ventolina, viento muy flojo, viento flojo, viento bonancible, viento fresquito, viento fresco, viento frescachón, viento duro, viento

10 Esta carencia ha quedado perfectamente subsanada para épocas anteriores del español gracias a la publicación del Nuevo Tesoro Lexicográfico del Español (s.XIV-1726), de Manuel Alvar Ezquerra y Lidio Nieto Jiménez.

11 Aunque la nómina de tecnicismos definidos es muy amplia, hay otros muchos que quedan sin precisar, y ello se debe, probablemente, a que el lector debía conocerlos gracias a su anterior obra: Manual de fïsica popular, a la que remite Gumersindo Vicuña en numerosas ocasiones. 
muy duro, temporal, borrasca y huracán (págs. 100), precisando que el viento frescachón se caracteriza porque es el que "tiende los trigos, y el muy duro rompe y desgaja las ramas, el huracán es capaz de derribar edificios y de arrasar cuanto encuentra a su paso" (págs. 100-101).

Además, con frecuencia, elige para las denominaciones de la realidad que describe voces en otras lenguas, es decir, opta por transcribir el préstamo en su lengua de origen y no en su forma castellanizada, como ocurre con caoutchouc por caucho, cirri y cirrus por cirro, cumuli por cúmulo, nimbus por nimbo o strati por estrato.

Finalmente, hay que señalar que Gumersindo Vicuña manejó y consultó el diccionario de la Academia, y así en la página 6 afirma que "este conjunto de fenómenos, la lluvia, los vientos, las tempestades, etc., constituye lo que vulgarmente se llama el tiempo. El Diccionario de la Academia española, en su última edición, da para esta palabra como acepción tercera, la siguiente: Cualquiera de las estaciones del año, primavera, estío, otoño e invierno [...]". A pesar de ello, el Manual de Meteorología popular recoge abundantes neologismos, esto es, voces o acepciones que no estaban presentes en la edición de 1869 del diccionario de la Academia, la última que había salido a la luz en ese momento, y que fueron incorporándose, o aún no lo han hecho, en sucesivas versiones del DRAE ${ }^{12}, y$, así, algunas de esas voces encuentran su primera documentación en la obra de Vicuña, de modo que ni centellómetro, ni evaporómetro, ni ozonométrico, ni pireliómetro, ni radiatorio, ni termógrafo, ni vexicular están presentes en repertorios anteriores ${ }^{13}$.

En este sentido, la presencia de neologismos en los trabajos y diccionarios científicos y técnicos de la época es muy abundante, ya que la maltrecha situación político-social de la España decimonónica "contribuyó a que la transferencia de conocimientos desde el exterior se acompañara del inevitable trasvase terminológico, a lo largo de todo el siglo, originando una queja continua por la entrada masiva de neologismos y la acuñación de términos nuevos" (Gutiérrez Rodilla, 1998: 80).

Algunos de los neologismos que aparecen en el Manual de Meteorología de Vicuña y su fecha de introducción en el DRAE, así como las definiciones de uno y otro, están recogidos en los siguientes cuadros:

\begin{tabular}{|l|c|}
\hline Voz & Fechade introducción \\
\hline caucho (caoutchouc), climatología, electrómetro, helióstata, higroscopio & 1884 \\
\hline \begin{tabular}{l} 
actinómetro, arsenito, higrométrico, psicrómetro \\
\hline actinometria, interplanetario
\end{tabular} & 1899 \\
\hline $\begin{array}{l}\text { barógrafo } \\
\text { isótera, isoquimena }{ }^{14}, \text { magneiómetro }\end{array}$ & 1925 \\
\hline $\begin{array}{l}\text { centellómetro, evaporómetro, ozonométrico, pireliómetro, radiatorio, } \\
\text { termógrafo, vexicular }\end{array}$ & $\begin{array}{c}\text { Primera documentación } \\
\text { de la voz }\end{array}$ \\
\hline
\end{tabular}

12 Para el tratamiento de los neologismos en el DRAE, véase M. Alvar Ezquerra (1992).

13 Ni Joan Corominas y José Antonio Pascual en el Diccionario crítico etimológico castellano e hispánico (1991), ni Lidio Nieto y Manuel Alvar Ezquerra en el Nuevo Tesoro Lexicográfico del Español (2007) las recogen.

14 Los adjetivos isótero, ra e isoquímeno, na entraron en el DRAE en 1899, pero los sustantivos no lo harían hasta época muy reciente. 


\begin{tabular}{|c|c|c|}
\hline$\underline{V o z}$ & Definición de G. Vicuña & Acepción del DRAE y fecha \\
\hline cirri & $\begin{array}{l}\text { Nubes más elevadas que están a } \\
\text { veces a } 6500 \text { metros de altitud. Son } \\
\text { trasparentes y blanquecinas. }\end{array}$ & \multirow{2}{*}{$\begin{array}{l}\text { Cirro. pl. Meteor. Conjunto de nubes que } \\
\text { tienen apariencia de zarcillos y se mantienen } \\
\text { en las regiones superiores de la atmósfera } \\
(1899)\end{array}$} \\
\hline cirrus & $\begin{array}{l}\text { Nube filamentosa que semeja las } \\
\text { barbas de una pluma. }\end{array}$ & \\
\hline cúmuli & $\begin{array}{l}\text { Nubes frecuentes en verano y que } \\
\text { semejan trozos de algodón con formas } \\
\text { redondeadas }[\ldots]\end{array}$ & $\begin{array}{l}\text { Cúmulo. Meteor. Conjunto de nubes propias } \\
\text { del verano, que tiene apariencia de montañas } \\
\text { nevadas con bordes brillantes (1899) }\end{array}$ \\
\hline nimbus & $\begin{array}{l}\text { Nube que toma un color oscuro } \\
\text { y aumenta en tamaño y falta de } \\
\text { trasparencia. Son signo casi seguro de } \\
\text { una condensación muy grande y, por } \\
\text { tanto, de una lluvia próxima. }\end{array}$ & $\begin{array}{l}\text { Nimbo. Meteor. Capa de nubes formada } \\
\text { por cúmulos tan confundidos, que presenta un } \\
\text { aspecto casi uniforme (1899) }\end{array}$ \\
\hline strati & $\begin{array}{l}\text { Nubes que forman capas paralelas } \\
\text { al horizonte e inmediatas a él, } \\
\text { predominando en la puesta del sol. }\end{array}$ & $\begin{array}{l}\text { Estrato. Meteor. Nube que se presenta en } \\
\text { forma de faja en el horizonte (1899) }\end{array}$ \\
\hline
\end{tabular}

Hechas estas precisiones, reproducimos a continuación el vocabulario técnico que se encuentra en el Manual de Meteorología popular de Gumersindo Vicuña, que, en la medida de lo posible y respetando siempre el contenido de la obra, hemos unificado para que todas las explicaciones muestren idéntica disposición formal - entre paréntesis van las páginas en que se encuentran-.

Actinometria, estudio de la acción luminosa medida por el actinómetro (53).

Actinómetro, conjunto de dos termómetros ordinarios encerrados dentro de dos tubos de vidrio, en los cuales se ha hecho vacío, uno con bola ennegrecida y otro con bola ordinaria; a veces se agrega un tercero con bola cubierta de una capa metálica brillante, para disminuir aún la acción radiatoria del calor de la luz; basta generalmente con los dos primeros (52-53).

Aerolito o meteorito, piedra que se coge en los campos caída del cielo y que es como residuo-sólido de las estrellas fugaces. Débense estos a la materia cósmica que hay en el espacio, y que al penetrar en la esfera de acción de nuestro planeta, cae atraída por él con tal velocidad que, al pasar por la atmósfera, se oxidan rápidamente sus elementos y arden, produciendo una vivísima luz, cayendo al suelo el núcleo sólido que no tuvo tiempo para combinarse con el oxígeno de la atmósfera $(174)^{15}$.

Anemómetro, aparato destinado a medir la velocidad y la dirección del viento [...]. El más sencillo de todos es la veleta, que marca dicha dirección y que es bien conocida (98).

Anotador o autógrafo, aparato meteorológico que marca las observaciones y señalan por medio de líneas sobre trozos de papel que luego se examinan despacio, con lo cual se consigue tener marcado el grado de cada instrumento en todos los instantes del plazo que

15 Aunque esta es la descripción más extensa de aerolito, en la página 14 aparece otra más breve, pero igualmente interesante: "Aerolitos o piedras meteóricas que vienen de los espacios interplanetarios, atraviesan la atmósfera, ardiendo a veces en ella, y caen sobre la corteza". 
se tarda en renovar el papel, cosa imposible para el observador más asiduo. Aumentan en gran número, de día en día cada vez más perfeccionados, si bien todavía presentan defectos y complicaciones tales que dificultan su vulgarización (11).

Apogeo, momento en que la luna está más lejos de la tierra (199).

Arco iris, [fenómeno que surge cuando] los rayos del sol, al refractarse en las gotas de agua de las nubes, sufren una descomposición en sus colores componentes. La mayoría de los rayos solares se reflejan en dichas gotas, y sólo una pequeña parte experimenta reflexiones interiores y refracciones, al menos capaces de producir el fenómeno óptico que estamos indicando. Los rayos rojos y los violados, que son los que tienen índice de refracción más separado son los más visibles, y en el intermedio se distinguen, con más o menos precisión, los rayos, también intermedios, del espectro solar. Para que el fenómeno se produzca, es necesario que el sol esté bastante bajo, pues si no, los rayos refractados no alcanzan a ningún observador colocado en la superficie de la tierra. Es preciso también que el observador esté de espaldas al sol, para que reciba la impresión de los rayos refractados. La forma circular del arco iris se debe a que es preciso un cierto ángulo, que dependen del índice de refracción y de la posición del sol y del observador, y todos los rayos refractados que forman el mismo ángulo con la recta paralela a la dirección primitiva de los rayos solares, que pasa por el ojo del espectador, formarán un cono, cuyo eje es dicha recta y cuyo vértice es el ojo (170-171).

Arrecife, vid. Corriente marina.

Atmósfera, envolvente gaseosa que gira con la tierra, cuya densidad disminuye a medida que consideramos sus regiones más altas, y cuyo límite de la parte eficaz se fijan en unos 70 kilómetros [...]. Lá atmósfera actúa como una caja: permite el paso con facilidad a los rayos rápidamente vibrantes del Sol, y no lo deja con la misma a los reflejados, refractados o difusos, que son como la consecuencia y desdoblamiento de aquellos [...]. La atmósfera tiene por misión, entre otras, no solo regularizar los cambios del calor, sino también dismi-

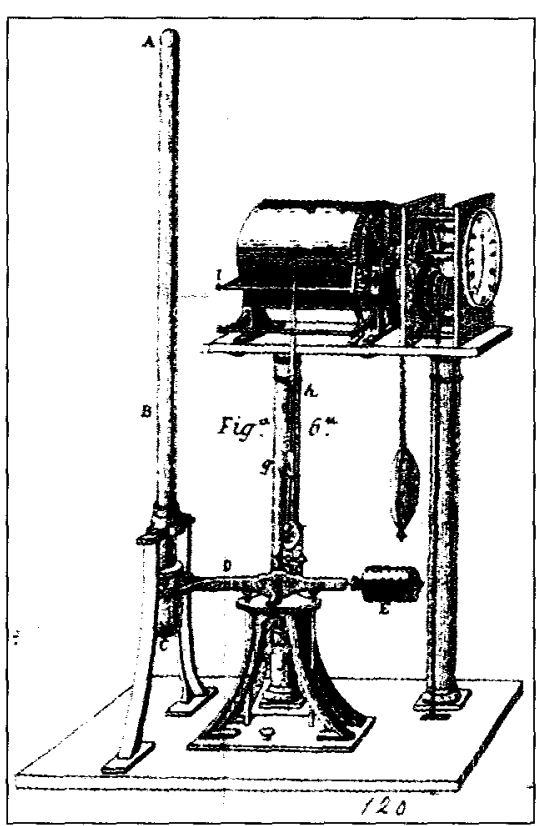

nuir el poder radiante de los rayos caloríficos $(20$, $21,23$ y 24$)$.

Aurora boreal, fenómeno muy frecuente en las regiones polares, visible a veces en nuestros climas y que es uno de los fenómenos más hermosos que nos presenta la naturaleza. Comienza por una luz cenicienta que se hace luego roja o violada, y que con inmensas llamas cubre gran parte del cielo. Suele empezar con un segmento de círculo que se va elevando y que se trasforma, empleando en esto a veces pocos minutos y otras más de dos horas. Los rayos constituyen magníficos cortinajes, y otras veces tiñen con un color casi uniforme una gran parte del cielo, produciendo un efecto sorprendente (173).

Autógrafo, vid. Anotador.

Barógrafo, vid. Barómetro-balanza.

Barómetro-balanza o barógrafo, aparato que sirve para medir la presión atmosférica [...]. Hay varios tipos: ordinario, de sifón y metálico (108 y 120; figura de la izquierda). 
Borrasca, acción rápida de vientos que azotan una extensión más o menos grande de terreno (163).

Brisas, vientos periódicos que se observan en la proximidad de las costas y cuya explicación es muy sencilla. Durante el día, los rayos solares calientan más los continentes que los mares, por efecto de la mayor capacidad calorífica del agua, y porque una parte del calor que afluye sobre el mar se emplea sólo en evaporar el agua, y por consiguiente, se produce un tiro sobre los continentes, y una llamada del aire que está encima del mar, formándose así una corriente fresca y húmeda, muy agradable en verano, época se su mayor intensidad, sobreviene pocas horas después de la salida del Sol (92-93).

Brújula, aguja imantada que tiene la propiedad de formar un cierto ángulo con el Meridiano terrestre. Esta propiedad ha servido y sirve aún a los navegantes para conocer la dirección del buque, toda vez que dicho ángulo determina fácilmente cuál es la traza del Meridiano terrestre en cada punto, y sirve de guía para la marcha del buque en el seno de los mares, sin tener que apelar a la posición del sol y de las estrellas [...]. Consta de una aguja imantada por su centro de gravedad sobre un estilete, en el cual puede girar, y colocada dentro de una caja con tapa de cristal, o en un sitio adonde no llegan las perturbaciones del viento (177).

Calor, es la forma, más común y conocida, de la energía natural. Cuanto mayor es la rapidez de esta energía mayor efecto produce en nuestro sentido del tacto, y a esta mayor o menor rapidez llamamos temperatura (35).

Capa invariable de la corteza terrestre, aquella en la cual la temperatura es constante desde ella aumenta casi uniformemente la temperatura a razón de un $\mathrm{I}^{\circ}$ por cada 30 metros, al menos en lo que se han profundizado (15).

Cartas magnéticas, cartas de mucho interés para la navegación, en las cuales se marcan las direcciones de los meridianos magnéticos, y también muy útiles para otros usos. $\mathrm{La}$ variabilidad de los números que representan las declinaciones hace necesaria la renovación frecuente de estas cartas. En vez de meridianos suelen trazarse las líneas que tienen igual declinación, o sea las que pasan por los puntos de la tierra, cuya declinación media es la misma (182).

Centella, vid. Rayo.

Centelleo de las estrellas, vibración, que suele observarse en las noches serenas, en la luz que nos mandan las estrellas, o sea los astros con luz propia, y que sirve precisamente para distinguirlas de los planetas (172).

Centellómetro, [aparato] ideado por Montigny, meteorólogo belga, destinado a medir el mayor o menor centelleo de las estrellas para cada posición de éstas en el cielo, pues varía el fenómeno con la mayor o menor capa atmosférica que han de atravesar los rayos que vengan de las estrellas. Pretende dicho físico servirse de este aparato para indicar la aproximación de la lluvia y aún de las borrascas (172-173).

Choque en retroceso, choque indirecto de un rayo sobre la tierra que produce efectos desastrosos (161).

Ciclón, nombre genérico que recibe el huracán que sigue cierta marcha circular jigantesca, que indica su movimiento rotatorio (93).

Cirri, nubes más elevadas que están a veces a 6500 metros de altitud. Son trasparentes y blanquecinas (147).

Cirrus, nube filamentosa que semeja las barbas de una pluma (147). 
Clima, conjunto de la acción meteorológica y terrestre de una localidad, y la importancia de su estudio no es sólo directa, sino también tiene por objeto servir para la predicción del tiempo [...]. Los climas superiores a la isoterma de $25^{\circ}$ se dicen abrasadores; cálidos los que corresponden a la zona comprendida entre las isotermas de 20 y $25^{\circ}$, como la isla de Cuba; apacibles a los que se hallan entre 15 y $20^{\circ}$, como sucede en la mayor parte de España; templados a los que están entre los 10 y $15^{\circ}$, como el Norte de España y Francia; fríos a los que se hallan entre 5 y $10^{\circ}$, como Inglaterra; y muy fríos a los que están entre 5 a $0^{\circ}$, como Noruega; denominándose glaciales los que caen bajo este último límite (214-215).

Clima abrasador, vid. Clima.

Clima apacible, vid. Clima.

Clima cálido, vid. Clima.

Clima continental, aquel en que hay bastante divergencia entre las líneas isóteras e isoquímenas y la línea isoterma. Se encuentra en la región central de la Península (216).

Clima frío, vid. Clima.

Clima glacial, vid. Clima.

Clima maritimo, aquel en que las líneas isóteras e isoquímenas no se separan mucho de la línea isoterma. Se encuentra en nuestras costas (216).

Clima muy frio, cfr. Clima.

Clima templado, cfr. Clima.

Climatología, ciencia que suministra reglas para la previsión del tiempo y da sanos consejos aplicables a la agricultura, a la navegación y a la higiene (8).

Coronas, dos o tres anillos coloreados y próximos entre sí y al sol, que ocupa su centro geométrico, que presentan el color rojo hacia el exterior. Débense a la difracción de los rayos solares en las gotas de agua de la atmósfera, y en la parte de la línea recta que va desde el radio al ojo del observador (171-172).

Corriente marina, diversa temperatura de las capas superficiales del mar, la cual a su vez influye sobre la temperatura de la atmósfera, los vientos, etc. [...]. Estas corrientes hacen posible el estado uniforme salino, o casi uniforme de las aguas del mar, en unos puntos se forman innumerables seres microscópicos unos y de tamaño apreciable otros, que mineralizan, por decirlo así, las sales disueltas en el mar, constituyendo arrecifes y montañas [...]. Realmente los vientos ejercen una acción poderosa en las corrientes marinas, pero no podemos entrar en detalles sobre este punto, puesto que aún no los hemos estudiado. El hecho cierto es, que por efecto de vientos regulares, de las otras causas citadas y de la inercia de la masa líquida, que una vez puesta en movimiento continúa en él con bastante regularidad, resultan las corrientes marinas $(30$ y 32$)$.

Cümuli, nubes frecuentes en verano y que semejan trozos de algodón con formas redondeadas; hállanse generalmente a menor altura que los cirri. Ordinariamente forman grandes masas y aumentan desde por la mañana hasta las tres o las cuatro de la tarde en que empiezan a disminuir, y a veces desaparecen con la puesta del sol; ganan altura durante las horas de calor y descienden en las frías. Débense estas nubes a las corrientes ascendentes del vapor de agua, y de aquí la explicación de sus principales variantes. La condensación de estas nubes determina las lluvias matinales en nuestros climas (147-148).

Declinación, ángulo que forman dos planos que pasen por el estilete [de la brújula] y sean verticales (177). 
Electrómetro, [aparato que sirve para medir] las energías eléctricas, ideado por el físico inglés Thomson. Consta de una caja de unos 3 decímetros de alto y 2 de lado en su base, cuyas cuatro paredes laterales son de vidrio, el fondo de madera sostenido por tres roscas, para poderlo colocar horizontalmente, y la tapa de caoutchouc endurecido; encima va un tubo de cristal de casi igual altura que la caja, en cuya parte superior hay un tamborcito, también de caoutchouc, del cual cuelga un alambre muy fino, de platino, que sostiene una chapa de aluminio cortada en forma de 8 y debajo un espejito (135-136).

Equinocio, época en la que los días son de igual duración y ocupan posiciones equidistantes en tiempo, el 21 de Septiembre y el 21 de Marzo, sin entrar en mayores detalles cosmográficos (209).

Escarcha, rocío helado, ya por la temperatura del ambiente, ya porque la tendencia a la evaporación absorbe calor [...], ésta puede producirse aunque el aire no esté a $0^{\circ}$, y no es extraño ver en la primavera, por la gran irradiación y rápido enfriamiento de las plantas, producirse escarchas sumamente perjudiciales para la agricultura (151).

Espacio interplanetario, parte pequeñísima, comparado con el infinito, del espacio en que se mueven los astros cuyos destellos nos alcanzan (16).

Espejismo, fenómeno de fácil explicación que proviene de la reflexión de la luz cuando se pasa del ángulo límite de la refracción (168-169).

Estrella, astro con calor y luz propios (16 y 172).

Estrella fugaz, luz vivísima, que suele verse durante las noches serenas, y sobre todo en las del mes de noviembre, correr por el firmamento y cuya rapidez es tal que se distingue un gran arco brillante (174).

Evaporómetro, [aparato que sirve para medir] la cantidad de agua evaporada directamente, para apreciar la parte de la lluvia que se pierde por este fenómeno. Esta evaporación al aire libre depende de la temperatura del líquido y del estado higrométrico del aire ambiente, así como de la acción de los vientos. Por esta razón, el vaso evaporatorio debe hallarse en un sitio despejado, para que se encuentre en condiciones análogas a las de las charcas y ríos. Generalmente, se usa con este objeto un sencillo vaso de hierro de forma cilíndrica y muy poca altura. Cada veinticuatro horas se mide el agua que queda, vertiendo el líquido del vaso e otro de vidrio bien graduado, que se llama probeta, y volviéndolo a llenar hasta una señal conocida que determina el volumen también conocido; la diferencia entre este y el que marca la probeta a las veinticuatro horas siguientes, al examinar el aparato, indica el volumen perdido por evaporación (75).

Evaporómetro anotador, aparato que consta de una báscula, cuyas oscilaciones de peso se marcan con un punzón en un cilindro dotado de un movimiento uniforme de rotación. Si sobre la plataforma de esta báscula se coloca el vaso evaporatorio se podrán apreciar las pérdidas en cada instante y con toda exactitud (77).

Grado higrométrico, vid. Higrómetro.

Granizo, [fenómeno que se produce por la] congelación de gotas de agua en el seno de las nubes, ya por una rápida evaporación que produce el enfriamiento, ya interviniendo además agentes eléctricos; cae en verano, y generalmente en las estaciones cálidas [...]. Se presenta generalmente después de las horas de más calor en cada día y sólo se verifica durante pocos minutos y acompañado generalmente de fenómenos eléctricos en el seno de la atmósfera [...]. Ordinariamente el granizo está formado de un núcleo mate, como de nieve apretada, rodeado de capas de hielo traslúcido, con las que alternan frecuentemente algunas 
opacas; en muchos casos hay rugosidades en la superficie y aún salientes y puntas [...]. El general es como guisantes, aunque en muchos casos adquiere el de garbanzos, y hasta él recibe el nombre de granizo [...]. La caída del granizo suele estar precedida de un gran ruido y crujido, al cual sigue casi inmediatamente el caer de las masas congeladas, y suele acompañar generalmente un fuerte viento y temporal (154-156).

Halos, anillos coloreados por el interior en rojo, y mayores que las coronas, que rodean también al sol. Débense a la refracción máxima de los rayos solares que atraviesan cristales de hielo situados en las regiones elevadas (172).

Helada, fenómeno de congelación del agua que hay en el terreno y en las plantas durante las noches frías (152).

Helióstata, aparato cuyo objeto es seguir el movimiento complejo del Sol por medio mecánico basado en los mismos principios que un reloj, el cual consigue que la pantalla quede siempre perpendicular al haz incidente (54-55).

Higrómetro, aparato destinado a medir la humedad del aire; como sus indicaciones no son muy seguras, se les denomina higroscopios por algunos [...]. El higrómetro más sencillo es el llamado de cabello. Consiste en un pelo de unos 2 decímetros de largo, que se ha hervido con carbonato de sosa para quitarle la grasa, el cual se sujeta por un extremo y se une por el otro a una palanquita que lleva una aguja, cuya otra punta, distinta de aquella a que se ata el cabello y muy larga, puede moverse sobre un arco de círculo graduado. Al contraerse el cabello hace mover la aguja en un sentido, y al dilatarse hay un pesito que la permite ir en el contrario, manteniéndose el cabello siempre tirante. Este aparato se gradúa introduciéndole en una campana, cuyo aire está perfẹctamente seco por sustancias químicas, que luego indicaremos, y se marca o en el punto a que llega la aguja al cabo de unas cuantas horas. Se introduce luego en otra campana donde hay trapos húmedos constantemente y se marca 100 en el punto donde permanece estacionaria la aguja al cabo de algún tiempo. Dividiendo en cien partes iguales el arco comprendido entre ambos puntos, se tienen los grados higrométricos (63-64).

Higroscopio, vid. Higrómetro.

Huracán, viento de tal velocidad que produce destrozos y desarreglos, y abrazan una extensión considerable sobre la superficie de la tierra (93).

Inclinación, ángulo que forma la aguja de la brújula con el plano horizontal (177).

Intensidad de la acción magnética, mayor o menor energía con que la aguja [de la brújula] tiende a ocupar una posición determinada, elemento que también es variable con la localidad y con el tiempo (178).

Linea isobara, la que pasa por los parajes que tienen la misma presión media (119).

Línea isoquimena, la que pasa por los puntos que tienen igual temperatura media en invierno (58).

Línea isótera, la que pasa por los puntos de la tierra que tienen igual temperatura media en verano (58).

Línea isoterma, temperatura media anual que comparten varios parajes de la tierra, sobre los que trazamos una línea sobre el mapa-mundi para que nos sirva de indicación de este hecho. Su nombre, de raíces griegas, quiere decir de igual temperatura (55-56).

Lluvia, producto general de la condensación de una nube, si bien a veces puede producirse ésta en gotas finas sin nube aparente (148).

Luz zodiacal, faja luminosa en el horizonte hacia el trayecto que aparentemente recorre el sol en el cielo, y que se nota al ponerse el sol en los meses de enero, febrero, marzo y 
abril, y poco antes de salir en el de noviembre [...]. Su color es de un blanco puro, y es un fenómeno de los más bellos que se notan con frecuencia en las zonas intertropicales, aunque no es tan común en la nuestra, donde adquiere un tinte rojizo (175-176).

Magnetómetro, aparato construido por Bruner, que sirve para medir la inclinación, la declinación y la intensidad de la acción magnética, y que tiene la ventaja de poderse trasportar cómodamente de un punto a otro (178).

Mar, gran masa de agua (27).

Marea, movimiento [del mar] debido a la acción de los astros (30).

Marea atmosférica, oscilación de la masa gaseosa por efecto de la atracción de la luna y el sol, de un modo análogo al que se ve en los mares (126).

Meridiano magnético, intersección del eje de la aguja [de la brújula] con la superficie terrestre (177).

Meteorito, vid. Aerolito.

Meteorología cósmica, influencia de los astros sobre los fenómenos que ocurren en el seno de la atmósfera y en sus inmediaciones a la costa terrestre. Esta hoy de moda y en tela de juicio (197).

Microscopio, poderoso auxiliar de la ciencia en nuestros días y que agiganta el sentido de la vista, permitiéndola examinar la forma y arreglo de los corpúsculos de diversa especie que flotan en el aire (142).

Niebla, condensación del vapor atmosférico en gotas sumamente pequeñas [...]. Las nieblas se producen generalmente durante la noche en los terrenos húmedos, sobre todo en los valles rodeados de montañas, pues entonces el enfriamiento es más rápido en la parte superior, y desciende el aire lamiéndolas y cargándose del vapor de agua del suelo (144-145).

Niebla seca, la producida por el humo acumulado sobre una población industrial, o por polvos de diverso origen arrastrados por vientos suaves, especialmente por las arenas de los desiertos y las cenizas volcánicas (145).

Nieve, cuerpo muy poco denso de agua congelada, que se presenta en el invierno para las altitudes ordinarias, pues claro es que en las superiores de las nieves perpetuas cae en todas estaciones (154).

Nimbus, nube que toma un color oscuro y aumenta en tamaño y falta de trasparencia. Son signo casi seguro de una condensación muy grande $\mathrm{y}$, por tanto, de una lluvia próxima (148).

Nube, niebla situada a mayor altura, procede a veces de ésta, o se forma como ella en ciertas regiones, aunque frecuentemente se debe al choque de dos vientos cargados de diversa cantidad de vapor de agua y a distinta temperatura, o bien al enfriamiento, y esto es lo más general, de un viento cuyo grado de saturación va aumentando por el enfriamiento sucesivo, debido al lugar que ocupa, hasta que llega a la verdadera saturación y se condensa su vapor (146).

Ozono, modificación del oxígeno de la atmósfera por efecto de la electricidad, habiendo cierta relación entre el mayor o menor desarrollo de los signos de dicho ozono y la energía eléctrica del ambiente (141).

Para-rayos, puntas metálicas de platino, por ser el metal menos oxidable y más difícilmente fusible, y un conducto metálico que llega hasta el fondo de un pozo para dar paso a la corriente eléctrica (162).

Perigeo, momento en que la luna está lo más cerca posible de la tierra (199). 
Piedra, granizo de mayor tamaño (155).

Piedra meteórica, vid. Aerolito.

Pireliómetro, aparato que consiste en un caja cilíndrica llena de agua y en la que hay un termómetro; una de las tapas de la caja está cubierta con una capa de negro de humo, con objeto de exponerla a la acción directa de los rayos solares, los cuales caldean el agua y determinan, al cabo de cinco minutos, por ejemplo, la cantidad de calor que transmiten dichos rayos. Con este aparato se han hecho los cálculos del poder calorifico del Sol (52).

Pluviómetro, aparato que se usa para medir el agua que en forma de lluvia cae sobre la superficie terrestre. Consiste generalmente en una caja de zinc en forma de embudo y cuya cantidad de agua se mide por una probeta $(80)$.

Pluviómetro anotador, el que consiste en un vaso situado a cierta altura y que comunica por un tubo con un depósito cerrado por la parte superior, en el cual hay un flotador terminado en una cremallera. Arriba está la cremallera, la cual engrana con una rueda, en cuyo eje horizontal hay además un excéntrico, sobre el que se apoya una varilla terminada en un punzón. El excéntrico está dispuesto de modo que hace subir la varilla a medida que asciende el flotador. Enfrente del punzón hay un cilindro vertical, provisto de un movimiento uniforme de rotación, dado por un aparato que está bajo el soporte y marca en él la curva indicadora del agua recogida (81-82; figura de la izquierda).

Pluviómetro totalizador, aquel que al llegar el agua cerca del embudo superior, se vacía por un sifón u otro medio, marcando en un contador el número de veces que se repite esta operación, y permitiendo así indicar la cantidad

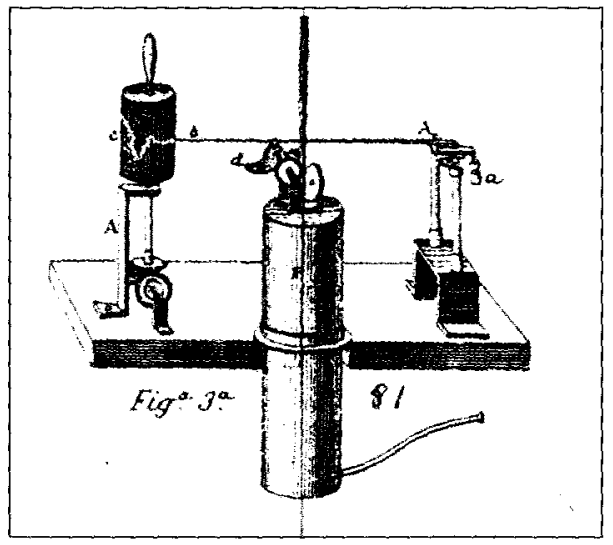
total de agua recogida al cabo de un tiempo cualquiera (81).

Potencial eléctrico, con este nombre se designa hoy el resultado de la suma de las acciones que ejerce la electricidad atmosférica sobre un punto en que nos fijemos; esta acción depende, como es consiguiente, del estado eléctrico general y de la distancia de los cuerpos cargados de electricidad que obran sobre dicho punto (138).

Probeta, vid. Evaporómetro.

Psicrómetro, aparato empleado para medir el estado higrométrico del aire. Consiste en un conjunto de termómetros, exactamente iguales, situados verticalmente uno cerca de otro, pero que el segundo tenía cubierto el depósito de mercurio con una telita constantemente humedecida [...]. El termómetro humedecido marcará menos temperatura que el seco, a causa del enfriamiento debido a la evaporación, y cuando más activa sea ésta, tanto mayor será aquél, de donde resulta que la diferencia de temperatura entre el termómetro seco y el húmedo da idea de la evaporación del agua, y, por tanto, de su fenómeno recíproco, a saber, la cantidad de vapor que hay en el aire (66).

Radiómetro, instrumento que consiste en una rueda de aspas oblicuas y cuyo diferente estado superficial (por su tersura) le hace ser más o menos sensible a la acción de la luz y girar con más o menos rapidez, dando así indicaciones de la intensidad de ésta (52).

Rayo o centella, descarga eléctrica entre una nube y la corteza terrestre (159). 
Relámpago, es la descarga entre dos nubes, asimilable a una gigantesca chispa eléctrica de una máquina, siendo el trueno como un chasquido colosal, repercutido por las nubes mismas. Suelen dividirse los relámpagos en tres clases: la primera consiste en un trazo vivo de luz, y es el más parecido a la chispa de la máquina eléctrica. Los de segunda clase son los más generales, y la luz se difunde y alcanza una gran superficie. A veces esto depende del sitio en que se halle colocado el observador. Los relámpagos de tercera clase son globos de fuego que atraviesan la atmósfera con una velocidad grande, pero muchísimo menor que la de los anteriores, que se dirigen a la tierra y estallan generalmente con un ruido infernal, produciendo destrozos si el estallido ocurre en un sitio habitado (159-160).

Relente, vid. Sereno.

Remolino o torbellino, [fenómeno que resulta] de un movimiento vertical del aire, debido a un enrarecimiento o a una contracción [...], pues entonces hay un verdadero concentramiento al bajar el fluido de una región que pudiéramos decir más ancha, puesto que el radio de la superficie es mayor a medida que idealmente nos alejamos de la tierra. Con los cambios de velocidad giratoria coinciden ascensos o descensos en el centro del torbellino (164-165).

Rocío, principio de la condensación del vapor de agua sobre las partes frías del terreno (150).

Sereno o relente, enfriamiento por irradiación que sobreviene poco después de ponerse el sol, y que, en los días tranquilos y despejados, produce un abundante rocío y un enfriamiento muy sensible para toda persona que lo sufra (153).

Solano, viento seco y abrasador que viene de África hacia España y es dañoso para la vegetación y los animales (92).

Solsticio, época en la que ocurren los días más largos y más cortos del año, el 21 de Junio y el 31 de Diciembre para nuestro hemisferio (209).

Strati, nubes que forman capas paralelas al horizonte e inmediatas a él, predominando en la puesta del sol (148).

Temperatura, vid. Calor.

Temperatura al aire libre, la obtenida en un sitio que no sufra el reflejo del calor por algún edificio u objeto próximo (39).

Tempestad, fenómeno producido por la acción mutua de energías originadas por la electricidad atmosférica [...]. El origen de éstas está en la aproximación de dos o más nubes entre sí, o a la superficie del terreno (159).

Termógrafo, termómetro que consiste en una cinta metálica, hueca y cerrada, de unos 30 centímetros de longitud y 20 centímetros cúbicos de volumen, completamente llena de alcohol, y retorcida alrededor de su eje; cuando por efecto del calor se dilata el líquido interior, destuerce algo la cinta, y tal pudiera ser la dilatación que la colocara recta, estallando luego si continuara; la presión interior no debe pasar de 8 atmósferas; lo contrario sucede al contraerse el líquido por el frío. En un extremo de la cinta hay una aguja que se une a él por un sencillo mecanismo: esta aguja tiene un movimiento alrededor de un punto fijo, debido a los movimientos de la cinta metálica, y por uno de sus extremos se apoya en un cilindro dotado de un aparato como el de un reloj. Si en dicho extremo hay un lápiz, y sobre el cilindro un papel, marcará en este una línea; es mejor que en el extremo haya un punzón, y que el papel esté ennegrecido con humo. Se gradúa este aparato comparando sus indicaciones con las de un buen termómetro ordinario. Supongamos que el cilindro dé una vuelta cada doce horas será preciso renovar el papel al cabo de este tiempo. Si la temperatura fuera constante, trazaría 
un círculo sobre el cilindro y al desarrollar el papel y extenderlo sobre una mesa, resultaría una línea recta; pero si, como sucede siempre, varía la temperatura en cada instante, dicha línea será helicoidal en el cilindro, y curva más o menos sinuosa en el desarrollo (37).

Tifón, huracán que ocurre en los mares de las Indias y que tantos destrozos ocasionan en nuestras ricas Islas Filipinas (93).

Torbellino, vid. Remolino.

Tormenta, conjunto de meteoros eléctricos y acuosos (163).

Tornado, nombre con que nuestros navegantes designan las grandes trombas, originadas por la tendencia a la rotación en los alisios en la zona que pudiéramos llamar clásica de las tormentas, o sea en la ecuatorial (166).

Tromba, ciclón pequeño, en el que verdaderamente no interviene de una manera sensible la electricidad, si bien a veces se produce esta forma de energía, como consecuencia del fenómeno (97).

Tromba, enorme torbellino de polvo que suele verse en los días tormentosos y que camina por el terreno desvastando cuanto encuentra y que eleva las aguas de los ríos, lagos y mares por donde pasa, poniendo en grave aprieto a los animales que encuentra en el terreno y a los buques con quienes tropieza en el mar; arranca y troncha los árboles, sobre todo los elevados, destruye las casas, destroza los campos y es motivo de terror y espanto para quienes ven llegar este meteoro (163-164).

Trueno, vid. Relámpago.

Vapor de agua, humedad que proviene de la evaporación continua de mares, lagos, ríos y vegetales que están esparcidos por todo el haz de la tierra. Esta humedad es necesaria para la economía animal, para la vida de las plantas, para la circulación del calor en la atmósfera y para llevar la lluvia fecundante a los terrenos elevados, desde los que baja por su propio peso a las llanuras para terminar en el depósito común, o sea el mar, produciendo con su juego un conjunto de fenómenos interesantísimos (59).

Vaso evaporatorio, vid. Evaporómetro.

Veleta, vid. Anemómetro.

Vexicular, estado en que cada elemento está compuesto de una película líquida llena de vapor de agua, lo cual forma un pequeñísimo globo flotante en la atmósfera, algo parecido a una pompa de jabón (148).

Viento, corriente, ora suave ora impetuosa y arrolladora, que resulta de los enrarecimientos en unos sitios, y estos originan en los inmediatos cierta llamada de aire, la cual se comunica a la próxima y así sucesivamente, producidos por las dilataciones de la atmósfera. Estos enrarecimientos o dilataciones, origen de los vientos, se deben en unos casos a la acción inmediata del calor, en otros a condensaciones o a producción de vapor de agua, ya a la acción inmediata en el primer sentido de las corrientes marinas, ya a la distinta repartición del calor y de la humedad entre los continentes y los mares. Ayudan también a la intensidad del viento la forma del terreno, los obstáculos, como las cadenas de montañas, y otros elementos de la superficie terrestre [...]. De todos modos, y sin salir de estas generalidades, podemos decir que la acción solar, por el desequilibrio del grado de calor o de la humedad, es la causa principal de los vientos, y que todo esto produce los cambios de la presión atmosférica que señala el barómetro. Los vientos producen graves perturbaciones en la atmósfera, la alteran y agitan, pero al propio tiempo mezclan sus elementos y modifican profundamente la acción de las demás condiciones de altitud, latitud, naturaleza del terreno y otras (24). 


\section{Conclusiones}

El léxico de la Meteorología que recopiló Gumersindo Vicuña pone de manifiesto la riqueza de un ámbito científico poco conocido hasta la fecha, expone los problemas, y las soluciones propuestas, con que se tropieza un científico al enfrentarse al vocabulario de especialidad, refleja la vasta formación de un especialista decimonónico y evidencia el interés por divulgar y popularizar la ciencia para que tuviese consecuencias en la vida diaria del país.

El Manual de Meteorología popular es, en definitiva, una buena fuente para conocer el caudal léxico de la ciencia, material indispensable para elaborar una historia de la lengua española.

\section{Referencias bibliográficas}

Ahumada Lara, I. (2000): "Diccionarios de especialidad en los siglos XVIII, XIX y XX", en Ahumada Lara, I. (ed.): Cinco siglos de Lexicografía del español. IV Seminario de Lexicografia Hispánica, Jaén, Servicio de Publicaciones de la Universidad de Jaén, 79-102.

Alvar, M. (1992): "Los diccionarios académicos y el problema de los neologismos", en Fundación Efe (ed.): El neologismo necesario, Madrid, Agencia EFE, 51-70.

Alvar Ezquerra, M. (1978): "Vocabularios específicos y vocabulario general", en Escobar, A. (ed.): Lingüistica y Educación. Actas del IV Congreso de ALFAL, Lima, Universidad Nacional Mayor de San Marcos, 164-177.

Alvar Ezquerra, M. (1989): "Los diccionarios con términos de la construcción", en Lexicografía descriptiva, Barcelona, Biblograf, 277-287.

Asclepio: archivo iberoamericano de historia de la medicina y antropologia médica (2003), 55 [coordinado por J. M. Blecua, J. Gutiérrea Cuadrado y J. A. Pascual].

Azorín Fernández, D. y M.a I. Santamaría (1998): "Los lenguajes de especialidad en los diccionarios del español de la primera mitad del siglo XIX", en Brumme, J. (ed.): La historia dels llenguatges iberoromanics d'especialitat (segles XVIT-XIX); solucions per al present, Barcelona, Universitat Pompeu Fabra, 359-378.

Bajo Santiago, F. (2002): "El nacimiento del léxico científico-técnico del vino en España: Esteban de Boutelou", en Echenique, M.a T. y J. Sánchez Méndez (eds.): Actas del V Congreso Internacional de Historia de la Lengua Española, vol. II, Madrid, Gredos, 1899-1914.

Battaner, P. (1977): Vocabulario político-social en España: 1868-1873, Madrid, Real Academia Española.

Battaner, P. (2001): "La traducción de los diccionarios de especialidad: estudio de algunos casos del siglo XIX", en Brumme, J. (ed.): Actas del II Coloquio Internacional de los lenguajes iberorrománicos de especialidad: la divulgación de la ciencia, Frankfurt am Main / Madrid, Vervuert / Iberoamericana, págs. 223-341.

Battaner, P. y L. Borrás (2004): “Traducciones y adaptaciones de diccionarios y obras de historia natural en el siglo XIX", en Alsina, V. y otros (eds.): Traducción y estandarización, Frankfurt am Main / Madrid, Vervuert / Iberoamericana, 161-191.

Cabré, M. T. (1993): La terminología: teoría, metodologia, aplicaciones, Barcelona, Ediciones Antártida.

Clavería Nadal, G. y J. Torruella (1998): "Els llenguatges d'especialitat en la lexicografia del segle XIX", en Brumme, J. (ed.): La historia dels llenguatges iberoromanics d'especialitat (segles XVII$X I X)$; solucions per al present, Barcelona, Universitat Pompeu Fabra, 327-340.

Contreras Izquierdo, N. (1997): "El léxico técnico en los diccionarios generales del siglo XIX: los términos de la Física en la $11^{\mathrm{a}}$ y $12^{\mathrm{a}}$ edición del Diccionario de la Real Academia Española", Acta Hispánica, 2, 43-67.

Contreras Izquierdo, N. (1998): "Las definiciones de sustantivos técnicos en dos diccionarios del siglo XIX", Acta Hispánica, 3, págs. 39-57.

Corominas, J. y J. A. Pascual (1991): Diccionario crítico etimológico castellano e hispánico, Madrid, Gredos, 6 vols. 
Fernández Clemente, E. (2002): "La recepción en España de la Segunda Recoluvión Industrial: las revistas de los ingenieros (1900-1936)", en Aubert, P. y J. M. Desvois (eds.): Les élites et la presse en Espagne et en Amerique Latine, des Lumières à la seconda guerre mondiale, Madrid, Casa de Velázquez, 1-21.

Gállego Paz, R. (2002): "Notas sobre la historia del léxico de la fotografía en español", en Echenique M. ${ }^{a}$ T. y J. Sánchez Méndez (eds.): Actas del $V$ Congreso Internacional de Historia de la Lengua Española, volumen II, Madrid, Gredos, 2051-2062.

García Godoy, M. ${ }^{a}$ T. (1995): El léxico del primer liberalismo español y mejicano (1810-1814): estudio de lexicología sociopolitica, Granada, Universidad de Granada.

Garriga Escribano, C. (1996-1997): "Penetración del léxico químico en el DRAE: la edición de 1817", Revista de Lexicografia, 3, 59-80.

Garriga Escribano, C. (1997): "La recepción de la Nueva nomenclatura química en español", Grenzgänge, 4, 33-48.

Garriga Escribano, C. (1998): "El Diccionario Universal de Física de Brisson (1796-1802) y la fijación lexicográfica de la terminología química en español", en García Turza, C., F. González Bachiller y J. Mangado Martínez (eds.): Actas del IV Congreso Internacional de Historia de la Lengua Española, Logroño, Publicaciones de la Universidad de La Rioja, 179-190.

Garriga Escribano, C. (2004): "Lengua y ciencia en español: reflexiones lingüística de los científicos en los siglos XVIII y XIX”, en Cabré, M. ${ }^{\text {a }}$ T. y R. Estopá (eds.): Objetividad cientifica y lenguaje, Barcelona, IULA / UPF, 183-193.

González Orejón, C. (2000): "Los nombres de flores y plantas en el Diccionario de Cuveiro", Revista de Lexicografia, 7, 63-75.

Güillén, Julio F. (1967): "El Diccionario marítimo español de 1831", BRAE, XLVII, 103-114.

Gutiérrez Cuadrado, J. (1996-1997): "La química y la lengua del siglo XIX: nota a propósito de pajuela, fósforo, mixto y cerilla", Revista de Lexicografia, 3, 81-93.

Gutiérrez Cuadrado, J. (1998): "Torres Muñoz de Luna y la lengua de la química en el siglo XIX", en Garcia, J. L. y otros (eds.): VI Congreso de la Sociedad Española de Estudios de las Ciencias $y$ de las Técnicas, volumen II, Segovia, Junta de Castilla y León, 701-711.

Gutiérrez Cuadrado, J. (2004): "Las traducciones francesas, mediadoras entre España y Europa en la lengua técnica del siglo XIX”, en Alsina, V. y otros (eds.): Traducción y estandarización, Madrid / Frankfurt am Main, Iberoamericana / Vervuert, 35-60.

Gutiérrez Rodilla, B. M. (1998): La ciencia empieza en la palabra. Análisis e historia del lenguaje cientifico, Barcelona, Ediciones Península.

Gutiérrez Rodilla, B. M. ${ }^{a}$ (1999): La constitución de la lexicografía médica moderna en España, La Coruña, Toxosoutos.

Gutiérrez Rodilla, B. M. (2000): "La higiene en la lexicografía médica de divulgación en la España decimonónica", Areas, 20, 51-60.

López Piñero, J. M. (ed.) (1992): La ciencia en la España del siglo XIX, Madrid, Marcial Pons.

Martín-Municio, Á. (1986): "Lexicografía de la ciencia y de la técnica", Telos: Cuadernos de Comunicación, Tecnología y Sociedad, 5, enero-marzo, 105-112.

Moya Cárcel, T. (2004): "La enseñanza de las matemáticas y el déficit científico español del siglo XIX", en Actas de las Jornadas de la Sociedad de Educación Matemática Al-Khwarizmi, Valencia, Sociedad de Educación Matemática Al-khwarizmi, 55-61.

Nieto Jiménez, L. y M. Alvar Ezquerra, (2007): Nuevo Tesoro Lexicográfico del Español (s. XIV-1726), Madrid, Arco/Libros, 11 vols.

Real Academia de Ciencias Exactas, Físicas y Naturales (1996): Vocabulario cientifico y técnico, Madrid, Espasa-Calpe, $3^{\text {a }}$ ed.

Revista de Investigación Lingüística (2004), volumen VII [coordinado por P. Díaz de Revenga y J. Perona].

Sala Caja, L. (2001): "La sinonimia en el vocabulario de la química del siglo XIX", en Brumme, J. (ed.): Actas del II Coloquio Internacional de los lenguajes iberorrománicos de especialidad: la divulgación de la ciencia, Frankfurt am Main / Madrid, Vervuert / Iberoamericana, 119-129. 